\title{
Über die Autoren/Abstracts
}

Beirat >Deutsch als Fremdsprache< des Goethe-Instituts

Dem Beirat >Deutsch als Fremdsprache des Goethe-Instituts gehören als stimmberechtigte Mitglieder an: Karl-Richard Bausch, Dagmar Blei, Konrad Ehlich, Anneliese Fearns, Claire Kramsch, HansJürgen Krumm (Vorsitz), Albert Raasch, Hans H. Reich, Günther Schneider, Inge Christine Schwerdtfeger, Dieter Wolff.

\section{Gerhard Bickes}

Dr. phil. (Heidelberg 1983). Nach dem Studium der Germanistik und Philosophie mehrjährige Lehrtätigkeiten (u.a. als DAAD-Lektor) an amerikanischen, britischen und australischen Universitäten. Seit 1989 im Lehrgebiet Deutsch als Fremdsprache an der Sprachlehranlage der Universität Mainz tätig, z. Zt. als akademischer Oberrat.

\section{Sylvia Bräsel}

Dr. phil.; Studium der Germanistik, Geschichte, Psychologie und Pädagogik an der Universität Leipzig; Literaturwissenschaftlerin und literarische Übersetzerin; Forschungsschwerpunkte: Literatur, Landeskunde, interkulturelle Prozesse; Lehrtätigkeit an deutschen, ostasiatischen und osteuropäischen Universitäten: von 1991 bis 1996 auf Vermittlung des DAAD in China und Südkorea tätig; zur Zeit als beurlaubte Hochschullehrerin des Bundeslandes Thüringen Fachberaterin für Deutsch im Auftrag des BVA/ ZfA in Sofia/Bulgarien.

\section{Angelika Braun}

Geb. 1954; Studium der Germanistik, Geschichte und Allgemeinen Linguistik in Mannheim. 1. und 2. Staatsexamen für das Lehramt an Gymnasien. Seit 1982 DaF-Unterricht und seit 1989 Tätigkeit in der DaF-Lehrer-Fortbildung, u.a. Deutsche Schule und Goethe-Institut MexikoStadt, Goethe-Institute in Deutschland, Universitäten Frankfurt und Mannheim, Volkshochschule Höchst. Seit 1994 als DAAD-Lektorin Leitung der Deutschabteilung des Instituts für Moderne Fremdsprachen an der Universität Guadalajara, Mexiko. Untersuchungen zu autonomiefördernden Unterrichtsformen und dem Einsatz der Neuen Medien (E-Mail-Tandem, WWW-Recherche) im Fremdsprachenunterricht.

\section{Georg Dietrich}

Geb. 1946; seit 1978 als Dozent am Goethe-Institut, 6 Jahre als Sprachabteilungsleiter am GI Bangkok, 3 Jahre Fachreferent am GI Prien, seit 1993 Sprachabteilungsleiter am GI Barcelona, Mitautor des Lehrwerks Sprachkurs Deutsch.

\section{Roland Grätz}

Geb. 1958; 4 Jahre als Deutsch-Fachleiter für die UNESCO und die Zentralstelle für das Auslandsschulwesen an Schulen in São Paulo, 1993 Dozentenausbildung am Goethe-Institut, seit 1995 am GI Barcelona als Sprachlehrer und in der Pädagogischen Verbindungsarbeit; zahlreiche Veröffentlichungen zur brasilianischen Literatur und Kunst im DaF-Unterricht.

\section{Hans Werner Hess}

Dr. phil.; Associate Professor für German / German Area Studies an der Hong Kong Baptist University. Seit 1994 Associate Course Leader des Europastudienprogrammes am Department of Government and International Studies. Spezial- 
gebiete: computerunterstütztes Lernen europäische und deutsche Landeskunde seit 1945. Weitere Informationen im Internet: http:/ / www.hkbu.edu.hk/ europe.

\section{Guido Rings}

Dr. phil.; geb. 1964 in Düsseldorf; Studium von Spanisch, Deutsch und Geschichte an der Universität Düsseldorf; 1. und 2. Staatsexamen in Spanisch und Geschichte sowie Diplom in Deutsch als Fremdsprache. Seit 1991 Dozent für Deutsch/ Wirtschaftsdeutsch und Neuere Deutsche Geschichte zunächst in Barcelona und Düsseldorf, später in Cambridge an der Anglia-University, 1996 Promotion an der Universität Trier. Publikationen in Deutsch als Fremdsprache, Spanisch und Geschichte mit Fokus auf Diskurstheorie und angewandte Linguistik.

\section{Dietmar Rösler}

Prof. für Deutsch als Zweit- und Fremdsprache an der Justus-Liebig-Universität Gießen. Zuvor Hochschullehrer am German Department des King's College London, Assistent am FB Germanistik der FU Berlin und DAAD-Lektor am University College Dublin.

\section{Gerhard Wazel}

Prof. Dr.; geb. 1939 in Falkenau (Sudeten); Studium der Germanistik, Anglistik, Pädagogik; Dissertation zum programmierten Grammatikunterricht, Habilitation zur Entwicklung des Sprechens bei ausländischen Studierenden der Germanistik. Lehrtätigkeit an Universitäten in Deutschland, Tschechien, Bosnien, Finnland, Österreich: Didaktik Deutsch als Fremdsprache, Angewandte Linguistik, Interkulturelle Kommunikation und Multimedia; Verfasser von Lehrmaterialien/Software für Deutsch als Fremdsprache.

\section{Bernd Wintermann}

Dr. phil.; Studium Germanistik/Geschichte in Tübingen, Berlin und Göttingen. 1972 bis 1983 und 1986 bis 1988 Lehrer für Deutsch als Fremdsprache am Studienkolleg Bochum. 1984 bis 1986 DAAD-Lektor an der Yonsei-Universität Seoul/Korea. Seit 1989 Leiter der Deutschkurse für Ausländer bei der Universität München. Seit 1995 Vorsitzender des Fachverbands Deutsch als Fremdsprache. 


\begin{tabular}{|c|c|c|}
\hline $\begin{array}{l} \\
\end{array}$ & 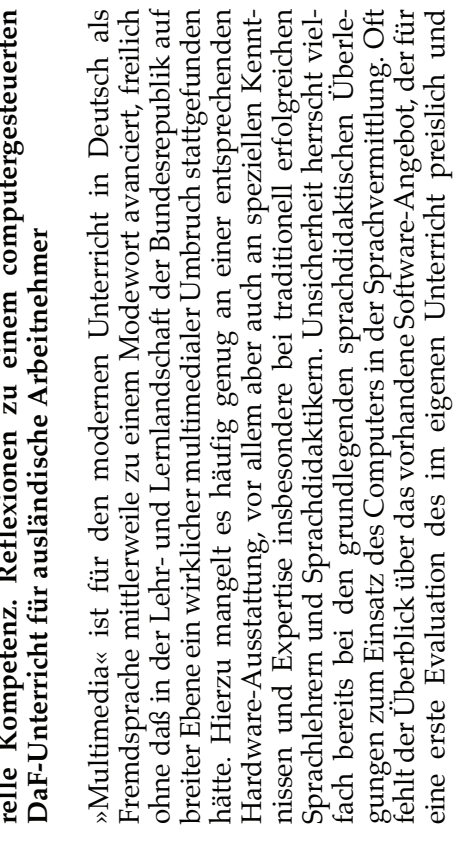 & 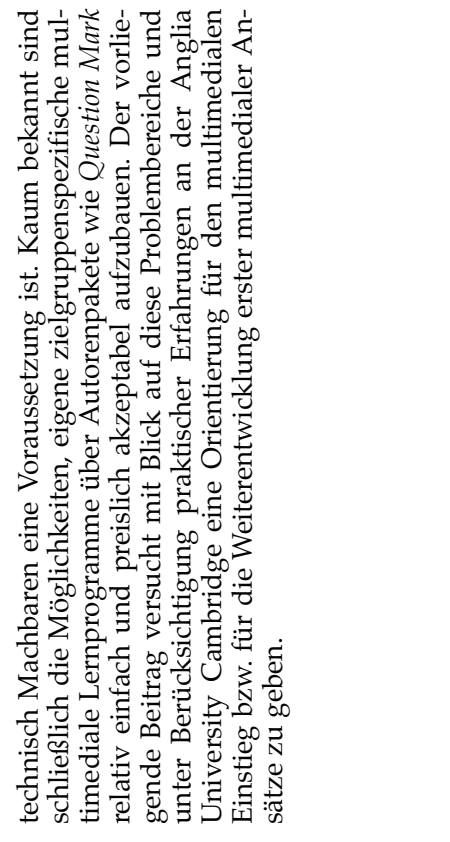 \\
\hline 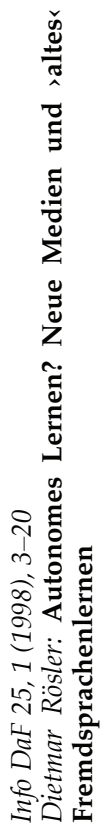 & 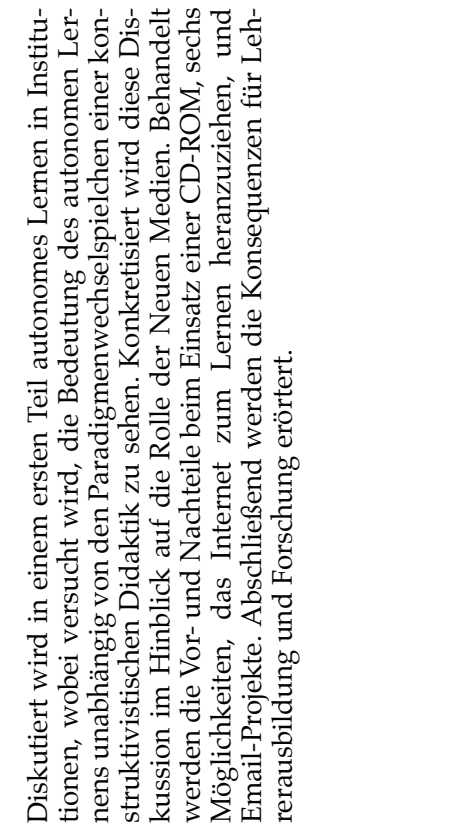 & 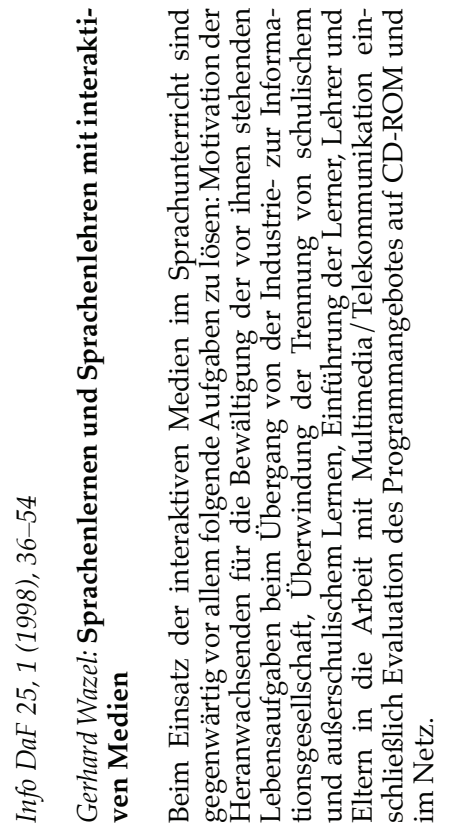 \\
\hline
\end{tabular}




\begin{tabular}{|c|c|c|c|c|}
\hline 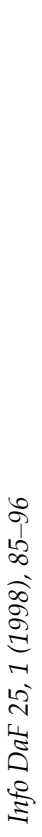 & 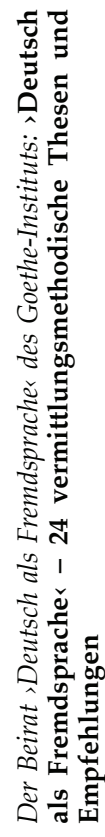 & 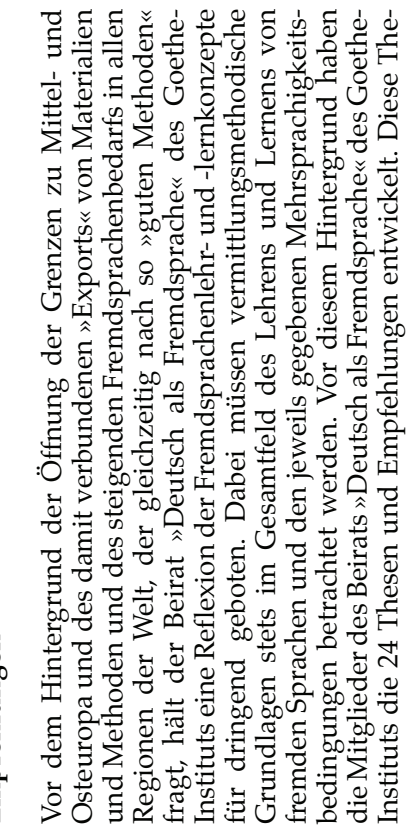 & 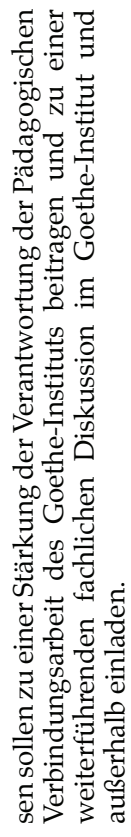 & \\
\hline 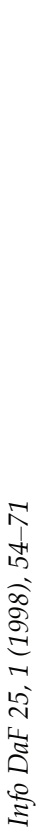 & 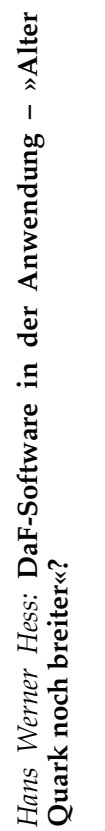 & 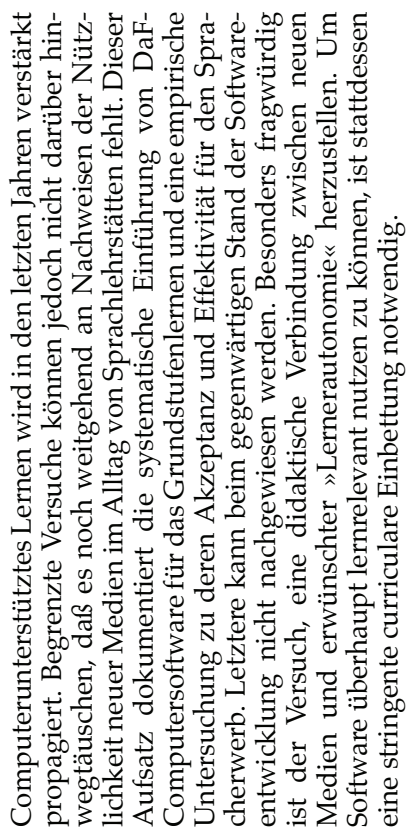 & 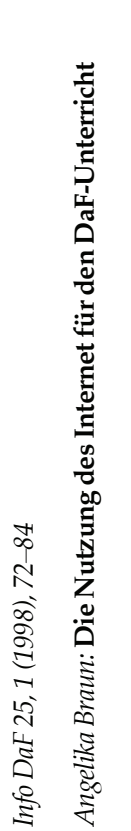 & 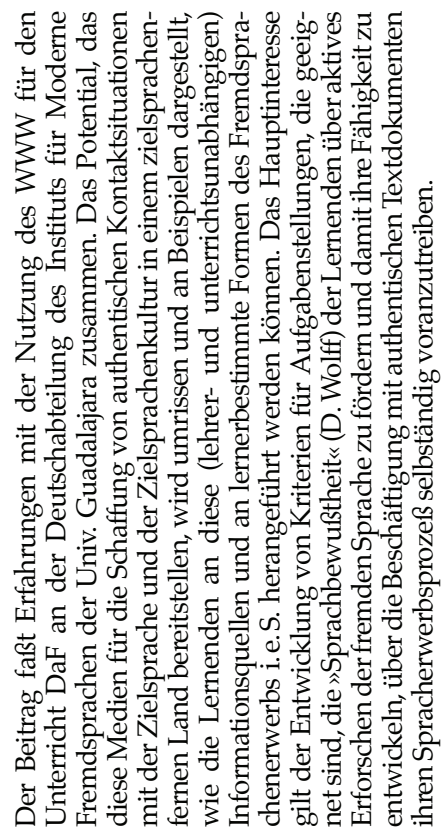 \\
\hline
\end{tabular}




\begin{tabular}{|c|c|c|c|}
\hline 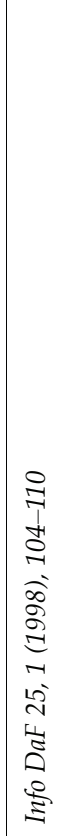 & 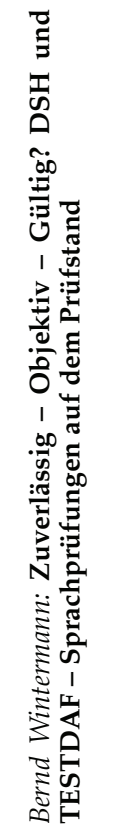 & 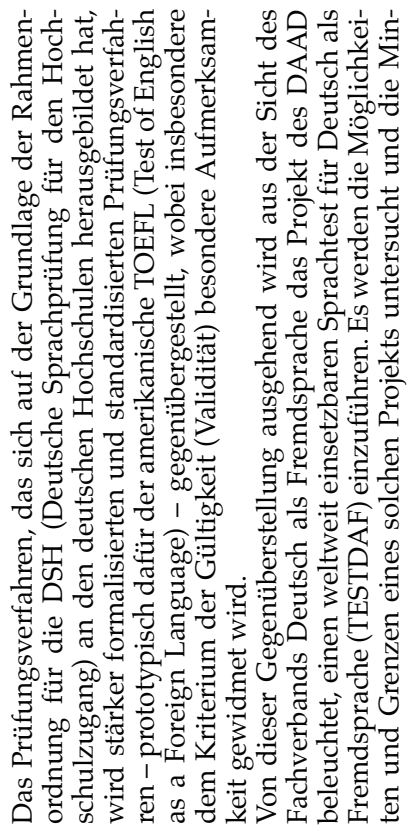 & 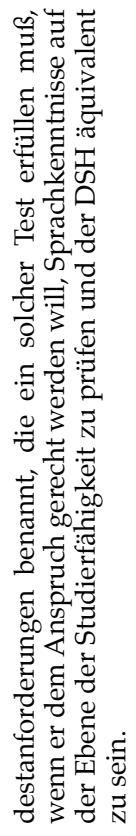 \\
\hline 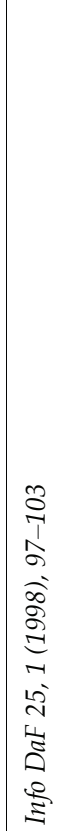 & 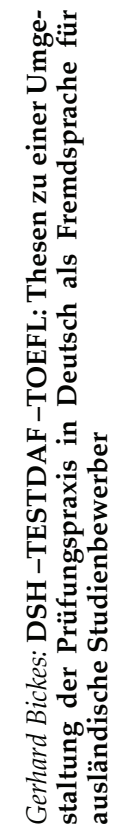 & 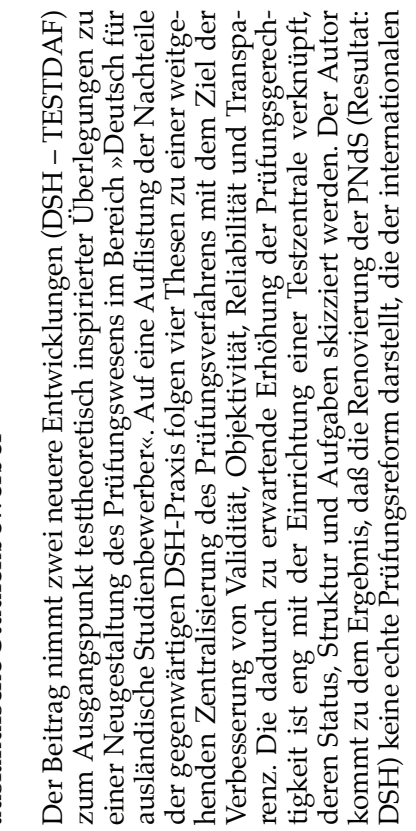 & 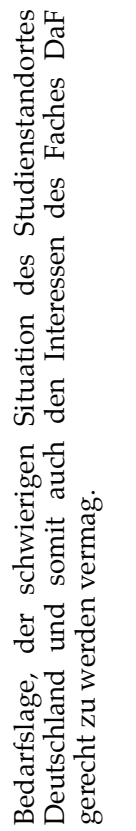 \\
\hline
\end{tabular}

